See discussions, stats, and author profiles for this publication at: https://www.researchgate.net/publication/327288606

\title{
Emergence of hidden phases of methylammonium lead iodide (CH\$_3\$NH\$_3\$PbI\$_3\$) upon compression
}

Article in Physical Review Materials · August 2018

DOI: 10.1103/PhysRevMaterials.2.085201

\section{CITATIONS}

6 authors, including:

José A. Flores-Livas

Sapienza University of Rome

54 PUBLICATIONS 1,718 CITATIONS

SEE PROFILE

Maximilian Amsler

Cornell University

74 PUBLICATIONS 1,787 CITATIONS

SEE PROFILE

Some of the authors of this publication are also working on these related projects:

interatomic potentials View project

Project

Theoretical studies on gas-phase biomolecules. View project
155

Daniele Tomerini

École Polytechnique Fédérale de Lausanne

14 PUBLICATIONS 134 CITATIONS

SEE PROFILE

8. Ariadni Boziki

École Polytechnique Fédérale de Lausanne

9 PUBLICATIONS 803 CITATIONS

SEE PROFILE 


\title{
Emergence of hidden phases of methylammonium lead iodide $\left(\mathrm{CH}_{3} \mathrm{NH}_{3} \mathrm{PbI}_{3}\right)$ upon compression
}

\author{
José A. Flores-Livas,,${ }^{1,}$ Daniele Tomerini, ${ }^{1}$ Maximilian Amsler, ${ }^{2}$ Ariadni Boziki, ${ }^{3}$ \\ Ursula Rothlisberger, ${ }^{3}$ and Stefan Goedecker ${ }^{1, \dagger}$ \\ ${ }^{1}$ Department of Physics, Universität Basel, Klingelbergstrasse 82, 4056 Basel, Switzerland \\ ${ }^{2}$ Laboratory of Atomic and Solid State Physics, Cornell University, Ithaca, New York 14853, USA \\ ${ }^{3}$ Laboratory of Computational Chemistry and Biochemistry, Institute of Chemical Sciences and Engineering, \\ Ecole Polytechnique Féderale de Lausanne, CH-1015 Lausanne, Switzerland
}

(Received 4 June 2018; published 29 August 2018)

\begin{abstract}
We perform a thorough structural search with the minima hopping method (MHM) to explore low-energy structures of methylammonium lead iodide. By combining the MHM with a forcefield, we efficiently screen vast portions of the configurational space with large simulation cells containing up to 96 atoms. Our search reveals two structures of methylammonium iodide perovskite (MAPI) that are substantially lower in energy than the well-studied experimentally observed low-temperature Pnma orthorhombic phase according to density functional calculations. Both structures have not yet been reported in the literature for MAPI, but our results show that they could emerge as thermodynamically stable phases via compression at low temperatures. In terms of the electronic properties, the two phases exhibit larger band gaps than the standard perovskite-type structures. Hence, the pressure-induced phase selection at technologically achievable pressures (i.e., via thin-film strain) is a viable route towards the synthesis of several MAPI polymorph with variable band gaps.
\end{abstract}

DOI: 10.1103/PhysRevMaterials.2.085201

\section{INTRODUCTION}

Perovskites are among the most promising and versatile class of candidate compounds for new or improved materials in energy applications, including photovoltaics, superconductivity, and lasing [1]. With the general formula $\mathrm{ABX}_{3}$, the perovskite structure consists of corner-sharing $\mathrm{BX}_{6}$ octahedra forming a three-dimensional (3D) framework that provides room for the A units in the resulting cuboctahedral cavities. Materials adopting the perovskite structure can display many desirable properties that are unparalleled among all other families of compounds [2-13]. This wide range of properties arises from the large number of elements that can be accommodated in the rather simple crystal structure. Further, the high-symmetry cubic perovskite structure is flexible and can readily distort, either as an effect of temperature and pressure, or of intercalation, doping, and defects [14], leading to orthorhombic-, hexagonal-, tetragonal-, or trigonal-type perovskites.

The lead-based methylammonium iodide perovskite (MAPI), with $\mathrm{A}=\mathrm{CH}_{3} \mathrm{NH}_{3}^{+}$, has a band gap of $\sim 1.55 \mathrm{eV}$ at room temperature, which is close to the optimal range of 1.1 to $1.4 \mathrm{eV}$ for a single junction photovoltaic cell [15]. Since its introduction as sensitizer for liquid-electrolyte-based dyesensitized solar cells in 2009 [15], MAPI has emerged as one of the most cost effective materials for photovoltaic applications, with continuously and rapidly improving efficiency. Rapid progress has been achieved within an extremely short period of time, and is a direct result of intensive studies [16] involving

\footnotetext{
*jose.flores@unibas.ch

†Stefan.Goedecker@unibas.ch
}

improved designs of the photovoltaic cell architecture, optimization of the preparation procedure, as well as modifications of optical and electronic characteristics through chemical substitution. Meanwhile, the power conversion efficiency of organic-inorganic halide perovskites in general has reached $23 \%$, approaching the Shockley-Queisser limit.

One of the most technologically relevant features of MAPI is its high optical absorption while incorporated in typical hybrid perovskite solar devices. In many of these devices, the MAPI absorbers are grown as homogeneous, ultra-thin films with a thickness of up to a few hundred nanometers, sandwiched between conductive layers. The strain arising (biaxial) at the interface between the MAPI and the substrate can distort the perovskite structure, and affect the optoelectronic properties. This has been shown for superlattices of $\mathrm{BaTiO}_{3}$, for which the interface structure enhances the polarization [17]. In turn, a careful selection of lattice mismatch to induce compressive and tensile strains has been proposed as an alternative route to tune the electronic properties of materials at interfaces without affecting the chemical composition. Although this could, in principle, work for perovskite materials and partially explain the degradation of electronic properties at interfaces, there is not solid evidence pointing to this effect.

The pressure-temperature phase diagram of MAPI is, however, rather complex. At ambient pressure and low temperature, MAPI adopts an orthorhombic structure with Pnma symmetry [18], which can be assigned the $a^{-} b^{+} a^{-}$label in Glazer notation [19]. At $162 \mathrm{~K}$, the Pnma phase transforms via a first-order phase transition involving a rearrangement of the octahedra to a tetragonal cell with $I 4 / \mathrm{mcm}$ symmetry, $a^{0} a^{0} c^{-}$, which persists at ambient temperature. Upon heating above $327 \mathrm{~K}[18,20]$, MAPI transforms to a cubic structure with $P m-3 m$ symmetry with the $a^{0} a^{0} a^{0}$ classification. 
Exploring the phase diagram of hybrid organic-inorganic perovskites under pressures is challenging, and available data points towards a complex enthalpy landscape rich in crystallographic transformations. Recently, state-of-the-art synchrotron powder x-ray diffraction experiments on MAPI under pressure were reported, however, without conclusively resolving the complete crystal structure [20].

Some of the experimental results disagree with each other on the nature of transformations occurring at moderate pressures. Szafrańki et al. [21,22] on one hand suggested that MAPI undergoes amorphization above $2.5 \mathrm{GPa}$. On the other hand, a transformation to a posttetragonal phase was observed by both Capitani and Jiang et al. [20,23]. A third set of experiments suggests that mixed organic-inorganic perovskites remain stable in their ambient (or a distorted) form and metalize for pressures above $50 \mathrm{GPa}[24,25]$. These discrepancies clearly show that further investigations are called for to conclusively map out the high-pressure phase diagram of MAPI.

One way to tackle this challenge is to use computational modeling based on first principles calculations in conjunction with structural search algorithms to explore potential highpressure phases. However, the modeling of MAPI using density functional theory (DFT) is challenging on its own. Standard exchange-correlation functionals used in DFT, such as the generalized gradient approximation, are known to underestimate the band gaps of MAPI, and structural features like the equilibrium volumes deviate from experimental values by up to $10 \%$. This discrepancy can be reduced by using improved functionals, e.g., by including empirical van der Waals (vdW) interactions. Further, spin-orbit effects are known to play a role in accurately reproducing experimental band gaps [26]. Despite these challenges, there have been several attempts to explore the chemical and structural landscape of perovskite materials, e.g., using approaches based on high-throughput schemes [27,28], and machine learning [29,30].

The most unconstrained manner to explore the potential energy surface (PES) is to employ a sophisticated structural search algorithm that samples the configurational space at a given stoichiometry. To the best of our knowledge, only Huan et al. [31,32] used this approach by employing the minima hopping method (MHM) [33,34] to directly explore the DFT PES of several halide perovskites (in particular, 12 atoms, or 1 f.u. for MAPI). This study reports interesting unobserved structures that are structurally related to the experimentally known, cubic high-temperature $\mathrm{Pm}-3 m$ phase. To correctly model the complex low-temperature orthorhombic phase (which has a significantly lower energy than the structures predicted by Huan et al.), a system size of at least 4 f.u. per cell (48 atoms per cell) is required to allow for different tilting angles of the octahedra. Since the complexity of the energy landscape grows exponentially with the number of atoms, a structural search directly at the DFT level with such a large cell is computationally prohibitive.

In this work, we address this issue by screening the PES of MAPI using a classical force-field to model large supercells in conjunction with the MHM. We identify two unreported polymorphs of MAPI that are considerably lower in energy than any of the perovskite (cubic, tetragonal, and orthorhombic) phases. Further, both structures are clearly thermodynamically favored at increased pressures
TABLE I. Effect of different exchange-correlation functionals on the lattice parameters and the volume of the orthorhombic (Pnma) structure at zero pressure. Experimental values stem from Ref. [18].

\begin{tabular}{lccccc}
\hline \hline Functional & $\begin{array}{c}a \\
(\AA)\end{array}$ & $\begin{array}{c}b \\
(\AA)\end{array}$ & $\begin{array}{c}c \\
(\AA)\end{array}$ & $\begin{array}{c}\text { Volume } \\
\left(\AA^{3}\right)\end{array}$ & $\begin{array}{c}\mathrm{T} \\
(\mathrm{K})\end{array}$ \\
\hline Exp. & 8.81 & 8.55 & 12.58 & 949 & 10 \\
Exp. & 8.86 & 8.57 & 12.62 & 960 & 100 \\
Exp. & 8.86 & 8.58 & 12.62 & 960 & 150 \\
LDA & 8.71 & 8.30 & 12.43 & 900 & - \\
PBE & 9.24 & 8.63 & 12.91 & 1031 & - \\
PBEsol & 8.99 & 8.45 & 12.61 & 959 & - \\
DFT-D2 & 8.68 & 8.57 & 12.60 & 939 & - \\
DFT-D3 & 8.91 & 8.54 & 12.75 & 971 & - \\
T-S & 8.97 & 8.49 & 12.73 & 972 & - \\
MB-d & 8.97 & 8.51 & 12.73 & 973 & - \\
dDsC & 8.96 & 8.52 & 12.71 & 972 & - \\
PBE0 & 9.03 & 8.65 & 12.93 & 1012 & - \\
SCAN & 8.95 & 8.62 & 12.73 & 983 & - \\
\hline \hline
\end{tabular}

and exhibit systematically larger band gaps than the know perovskites.

The paper is organized as follows. Section II consists of three subsections: the first, Sec. II A, summarizes the structural search and discusses the thermodynamic stability of the lowenergy phases. Section II B investigates the effect of pressure and stability upon compression, while Sec. IIC assesses the electronic properties. Finally, Sec. III discusses potential routes towards the synthesis of the new phases of MAPI.

\section{RESULTS}

\section{A. Novel phases of MAPI}

We use a classical interatomic potential parametrized by Handley and Freeman [35], as implemented in the General Utility Lattice Program (GULP) molecular modeling package [36]. GULP was coupled to the MHM to screen the PES using simulation cells containing 2, 4, and 8 units (i.e., 24, 48, and 96 atoms). More than 140,000 polymorphs of MAPI are generated using this procedure. To validate the accuracy of the force-field, we randomly select 250 structures and optimize them using KS-DFT with LDA and PBE functionals. We find a remarkably good correlation between the DFT and force-field energies (see Supplemental Material [37]), which justifies its use for a reliable prediction of low-energy structures.

To assess which exchange-correlation functional is best suited to model MAPI, we compute the lattice parameters and cell volumes of the experimental ground state, the orthorhombic phase with 48 atoms, using a set of different functionals (see Table I). We employ three popular standard functionals: the local-density approximation (LDA) [38] and two generalized gradient approximations (GGA) (PBE [39] and PBEsol [40]). We also assess functionals that (empirically) take into account vdW dispersion, namely the DFT-D2 method [41] and the zero damping DFT-D3 method of Grimme [42], the TkatchenkoScheffler (T-S) method [43], and the many-body dispersion (MB-d) energy method [44], and the dispersion correction (dDsC) method [45]. We also investigate a hybrid functional 


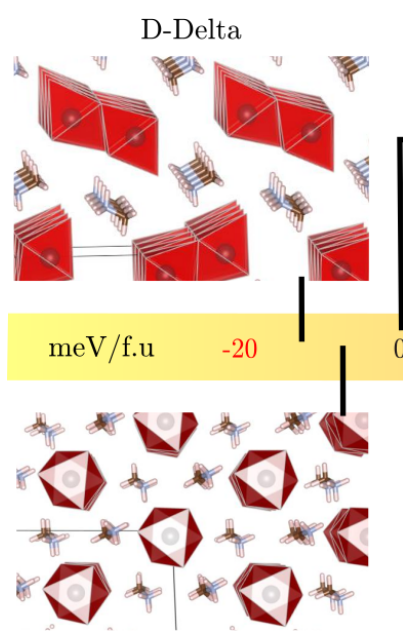

Delta

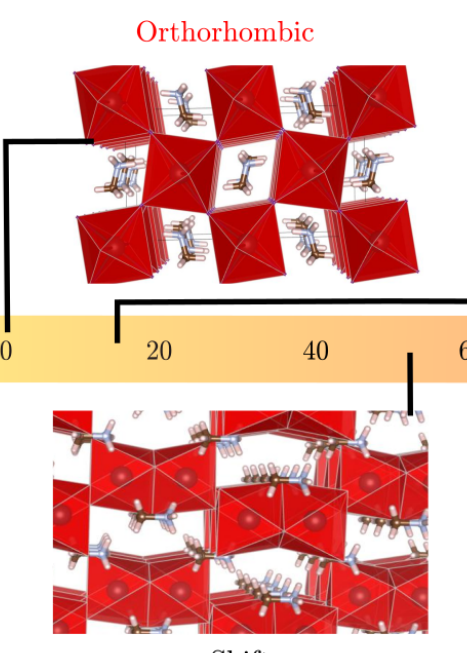

Shift

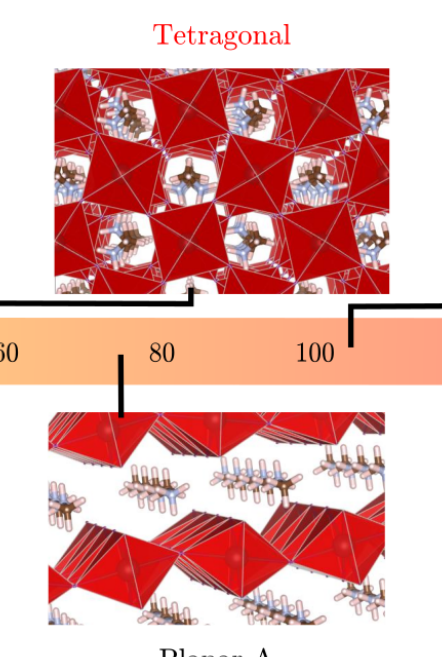

Planar A

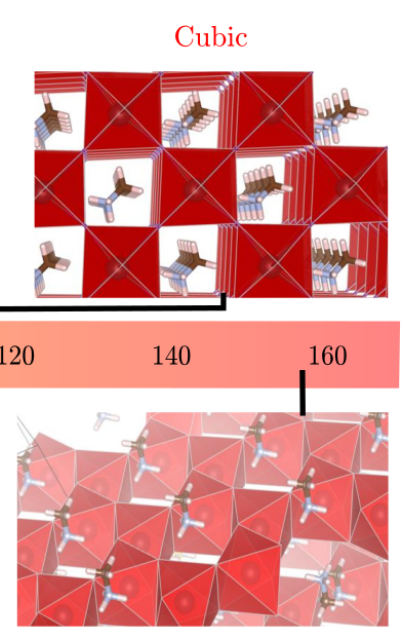

Planar C

FIG. 1. Energy ranking of various MAPI polymorphs with respect to the orthorhombic (Pnma) phase with DFT using the meta-GGA $(\mathrm{SCAN}+\mathrm{SOC})$ functional. Structural motifs include the known perovskites (cubic, tetragonal, and orthorhombic) as well as the low-lying polymorphs predicted in this work.

which includes a fraction of exact-exchange, PBE0 [46], and the recently developed strongly constrained and appropriately normed (SCAN) meta-GGA functional [47].

As expected, the standard Kohn-Sham functionals provide a fair description of the lattice constants when compared to experiments $[48,49]$. LDA and PBE under and overestimate the lattice constants, respectively, and PBEsol, while at first glance provides cell volumes in excellent agreement with experiment, exhibits a deviation of the individual lattice components. Empirical inclusion of $\mathrm{vdW}$ interactions greatly improves the unit cell volumes (D2 and D3) [50]). The D2 and D3 approximations show $b$-components in excellent agreement with experimental values [51]. All vdW methods lead to very accurate $c$-component when compared to experiments. Surprisingly, the inclusion of exact-exchange via hybrid-DFT leads to a systematic overestimation of the three lattice parameters, while being the computationally most costly method studied here. Finally, our analysis indicates that the metaGGA SCAN functional improves the overall description with respect to experimental values compared to other semilocalbased functionals. It is worth mentioning that the volume obtained by SCAN is reliably close to the experimental ones [52-54] even without the inclusion of vdW correction (and will become relevant under pressure), and represents a good trade-off between accuracy and computational cost. Therefore, henceforth we will use the SCAN functional for all structural and thermodynamic evaluations.

First, we compute the thermodynamic stability of the various polymorphic modifications of MAPI based on the SCAN functional. We identify several distinct structural motifs within a small energy range around the ground state phase which we consider in our analysis. Figure 1 shows representative structures of various low-energy phases, ranked according to their energies with respect to the orthorhombic structure of MAPI. We use our recently developed structural fingerprint $[55,56]$ to classify the large amount of distinct phases into families of similar structural motifs (see SI). One family that we call "planar" encompasses all structures with layered octahedra that share edges and are intercalated with molecular units of
$\mathrm{CH}_{3} \mathrm{NH}_{3}^{+}$between these planes. The structure referred to as "planar A" lies between the tetragonal and the cubic phase with an energy of $\sim 75 \mathrm{meV} / \mathrm{f}$.u. above the ground state, while "planar C" lies at an energy scale of $\sim 125 \mathrm{meV} / \mathrm{f}$.u. ("planar B" is not shown in the figure). Considering the energy range spanned by the experimentally observed cubic and orthorhombic phases of $\sim 105 \mathrm{meV} /$ f.u., "planar C" is unlikely to be observed in experiments, while "planar A" lies readily within the range of the thermodynamic scale of metastable phases and is hence potentially accessible $[57,58]$.

Another structural motif that we find and define as the "shift" family consists of $\mathrm{PbI}_{6}$ octahedra arranged in $(c h)_{4}$ fashion according to the $h-c$ notation $[59,60]$. These phases have energy differences with the ground state that are at least twice that of the tetragonal phase, but lower than the cubic phases. The organic components are arranged in a way as to compensate for the change in the back-bone structure of the perovskite-type phase. Hence, this shift type of structural motif could be energetically further enhanced if other types of cations are selectively used. Other arrangements of hexagonal and cubic layers such as $(c c h h)_{2}$ were found in our force field search, but not included in further analysis as they were found to be energetically higher.

Finally, and most significantly, we report here the discovery of two classes of polymorphs which lie -15 and $-9 \mathrm{meV} /$ f.u. lower in energy than the reference orthorhombic phase, namely the double-delta (D-Delta) and delta structures. The unit cell of the delta structure contains 48 atoms, and consists of face-sharing octahedra stacked on top of each other to form pillars, while the organic units uniformly surround them. In this structure, the $\mathrm{Pb}$-I distance within the octahedral arrangements is on average $3.25 \AA$, and the distance between the pillars and the molecules are on average $3.6 \AA$. The D-delta phase also has a pillar-like structure, but the octahedra share edges instead of faces. We selected three structures (that we labeled $-\mathrm{A}: 48$ atoms and $-\mathrm{B}$ :, $-\mathrm{C}$ : 96 atoms) with different orientation of the methylammonium molecules; the differences are shown in the supplementary information. The average $\mathrm{Pb}$-I distance in D-delta is $3.27 \AA$, and the distance from the "double-pillars" to 

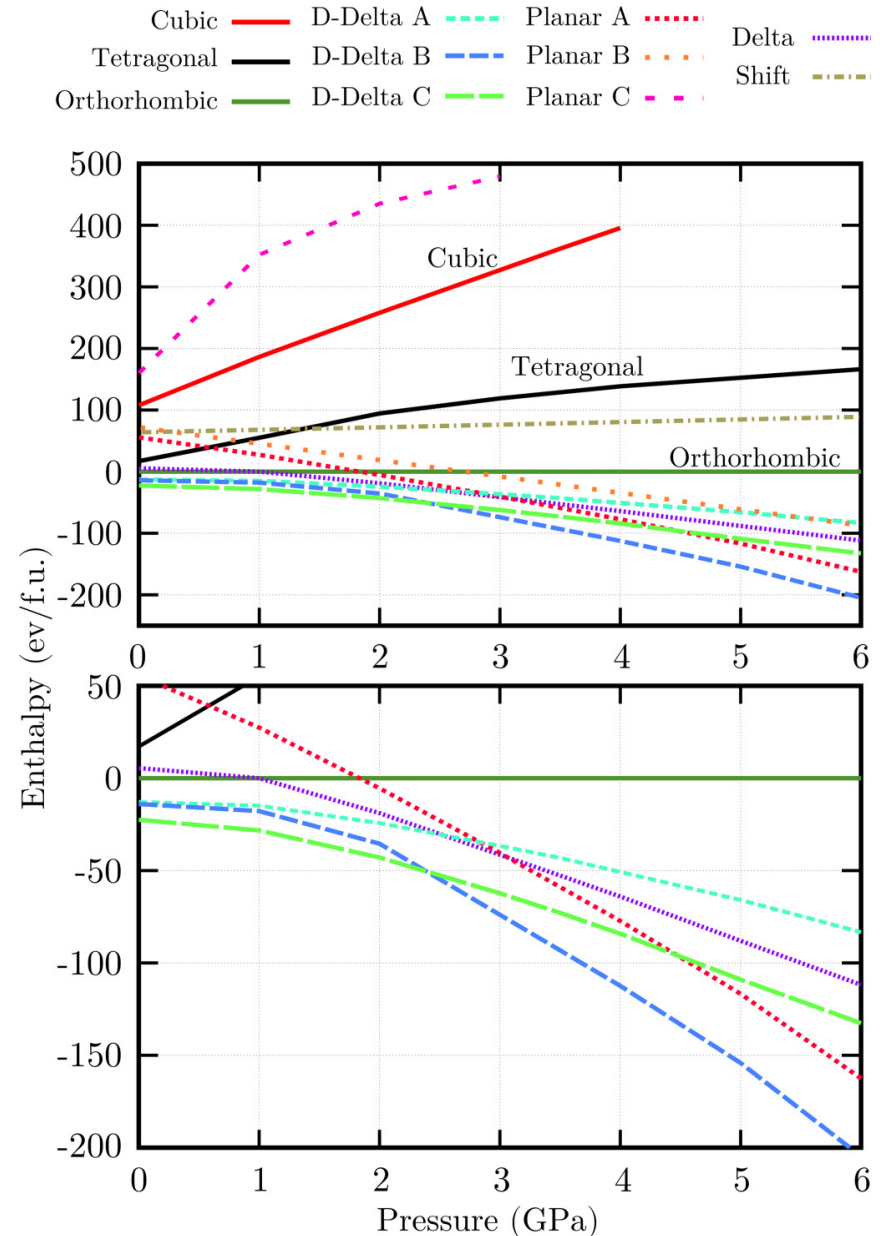

FIG. 2. Calculated formation enthalpies per formula unit (12 atoms) of various competing MAPI polymorphs as a function of pressure, computed using the meta-GGA functional (SCAN + SOC). Bottom panel is a magnification of the low-enthalpy region. All enthalpy differences are given with respect to the orthorhombic (Pnma) phase.

the molecules is on average $3.7 \AA$. The coordination number per octahedra (i.e., the number of iodide ions linked to $\mathrm{Pb}$ centers of the octahedra) is nominally close to 6 in both structures (delta and D-delta). Note that in all the structures described here, all molecular $\mathrm{CH}_{3} \mathrm{NH}_{3}^{+}$remain intact: the interatomic distances and the overall conformation are preserved. The main structural differences between the structures arise from the different octahedral conformations and the rearrangement of molecular cations.

\section{B. MAPI under pressure}

We next investigate how pressure affects the enthalpy and volume of the experimental and predicted phases of MAPI with respect to the orthorhombic phase, and we report the results in Fig. 2. Clearly, the tetragonal and cubic phases are greatly destabilized upon compression, while other phases such as planar A and B and the delta phases become enthalpically preferable over the orthorhombic phase. Within the range of pressure that we study here we find that the delta phases, in particular the D-Delta structures, are predominantly stabilized

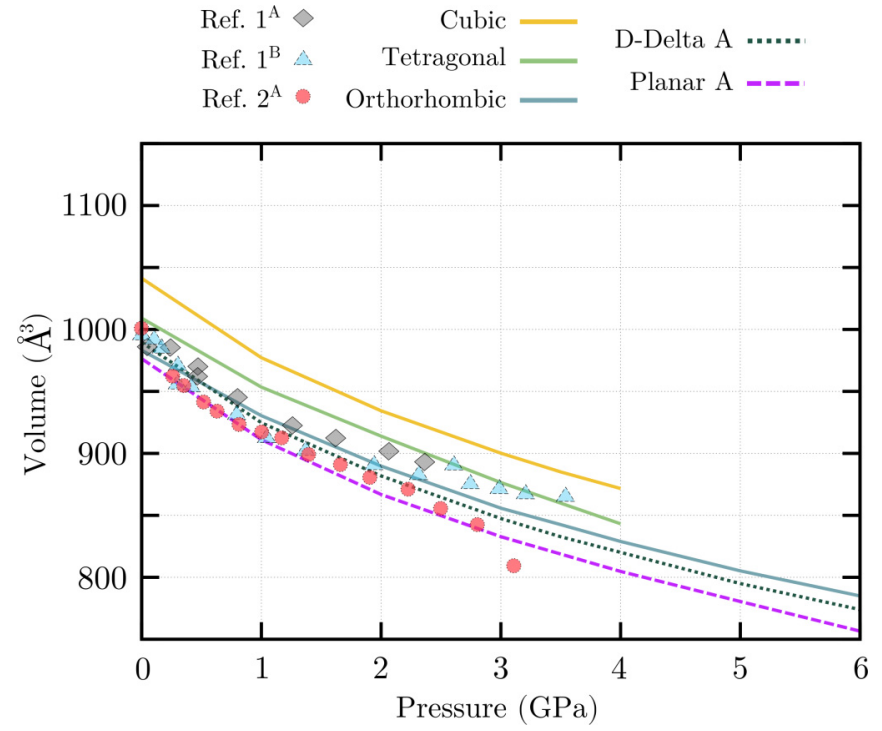

FIG. 3. Pressure-volume dependence for various predicted polymorphs of MAPI and experimental values from Refs. [20,23].

upon compression. More specifically, we note that several D-Delta phases, which all contain distinctly oriented organic cations, leave the inorganic framework completely intact upon compression. The D-Delta C phase is thermodynamically stable between zero and $2.5 \mathrm{GPa}$, above which the D-Delta $\mathrm{B}$ structure is favored. This phase exhibits a more symmetric arrangement of the organic moieties, and persists as the thermodynamic ground state up to at least $6 \mathrm{GPa}$. Both the $\mathrm{B}$ and $\mathrm{C}$ phases are members of the double-delta family, which appears as the most stable class of structure at all pressures. Therefore it appears that the face- and edge-sharing arrangement of the octahedra, being more compact, is favored over a less compact, corner-sharing arrangement. The enthalpy differences between the distinct double-delta phases (A, B, and C) primarily stem from different orientations of the molecular parts in the space between the octahedral frameworks, which interact primarily through different dipole arrangements. A clear enthalpic preference simply based on the relative unit cell volumes is therefore not possible.

In Fig. 3, we map out the pressure dependence of the atomic volumes of the various predicted polymorphs of MAPI and compare them to the experimentally available data. The cubic phase has the largest volume of all experimentally observed perovskite structure types, followed by tetragonal and orthorhombic as more compact phases. The room temperature compression carried out by Jiang et al. [23] reported the volume for the tetragonal perovskite (see Supplemental Material XRD section [61]) and its partial transformation to a Im-3m structure above $0.4 \mathrm{GPa}$. Both phases coexist with each other up to $2.5 \mathrm{GPa}$, and at $2.7 \mathrm{GPa}$ an additional phase appears, whose crystal structure could not be determined. The authors, however, proposed an indexing with the Immm [23,62,63] space group. Finally, the onset of amorphization occurs at pressures exceeding $4.7 \mathrm{GPa}$.

Capitani et al. [20,64] also reported a similar pressurevolume curve as in one of the experiments Jiang et al. (1B in the figure), but with a clear deviation above 2 GPa towards smaller volumes. The authors interpreted this effect as the 


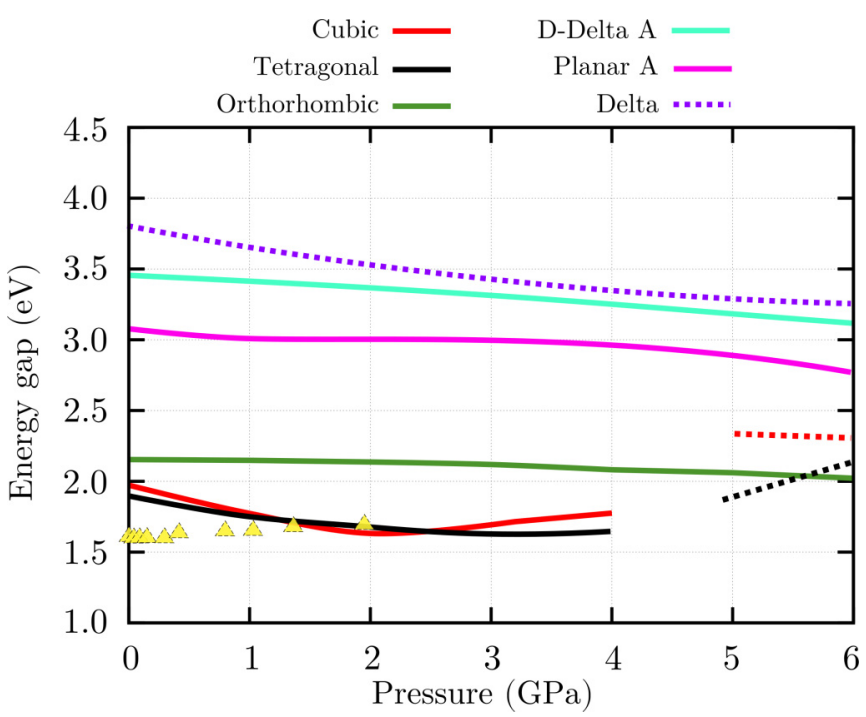

FIG. 4. Electronic band gaps calculated with the hybrid functional PBE0, incorporating spin-orbit effects (SOC) on optimized geometries from meta-GGA for known and predicted phases of MAPI under pressure. Direct band gaps are depicted as solid lines, while dashed lines denote indirect band gaps. The yellow dots represent experimental (photoluminescence) values from Ref. [23].

emergence of amorphization, a reversible process that leads to a recrystallization of the orthorhombic Imm 2 upon pressure release. However, from the computed volumes of our predicted phases one can see that the volume of planar-A, delta and double-delta, are all smaller than the one of the orthorhombic (perovskite) phases due to the optimized conformation of octahedral sharing. Hence, these phases serve as potential candidate phases that would explain the experimental results of Capitani et al.

\section{Electronic structure}

We characterize the known and predicted phases in terms of their electronic properties under compression. In the past, multiple studies have shown that, to have a correct description of the electronic properties of MAPI from first principles, it is important to take into account spin-orbit coupling (SOC) $[49,51,65]$. The calculated GGA-PBE scalar-relativistic band gaps of MAPI polymorph (tetragonal at experimental volume) is on average close to $1.6 \mathrm{eV}$, a value that is coincidentally very close to the experimental value of $1.682 \mathrm{eV} \mathrm{[66].} \mathrm{This} \mathrm{is}$ an artifact due to the neglect of SOC of the $\mathrm{Pb}$ and I atoms in the calculation $[51,65]$. When SOC is taken into account, the calculated band gap within fully relativistic DFT is $\sim 0.6 \mathrm{eV}$ (see Supplemental Material [61]). To address this issue, we use the relaxed geometries calculated with meta-GGA (SCAN) and perform single-point calculations with hybrid-DFT (PBE0) including SOC to accurately estimate the electronic band gaps (see benchmarks and details in Supplemental Materials [61]).

Figure 4 shows the evolution of the calculated band gaps under pressure for the three know polymorphs and selected predicted structures of MAPI. Most of the structures have direct transitions (optical band gaps), which are shown as solid lines, while those with indirect gaps are shown with dashed lines. Experimental photoluminescence measurements of the band gaps under pressure are included for the tetragonal phase [23]. The experimental values reported in the literature at ambient pressures for the three perovskite polymorphs are scattered between 1.55 to $1.72 \mathrm{eV}$ [66-73]. We assign this large spread to several potential sources, (a) to the range of temperature at which the polymorphs are reported to coexist [18], (b) to different orientations of the methylammonium units (leading to changes of the band gap within a few tens of meV), (c) due to errors arising from the method of the measurements (optical absorption edge, photoluminescence, electrical measurements), and (d) to the macroscopic conformation of the sample (single crystal of polycrystalline heterojunctions). According to our calculations, the Delta phase has an indirect band gap of $3.81 \mathrm{eV}$ at zero pressure, which decreases to $3.26 \mathrm{eV}$ at $6 \mathrm{GPa}$. The double-delta "A" phase has a direct band gap of $3.45 \mathrm{eV}$ at zero pressure that decreases to $3.12 \mathrm{eV}$ at $6 \mathrm{GPa}$. The distinct alignment of the organic components in the double-delta phases can affect the electronic band gap and change its magnitude by up to $100 \mathrm{meV}$.

\section{DISCUSSION}

First, let us address in more detail whether the predicted, new phases of MAPI can be synthesized. As previously described, the known structural transitions in hybrid perovskites at ambient pressure are driven by temperature. Below $132 \mathrm{~K}$, the tetragonal phase transforms to the low-temperature orthorhombic phase, which persists at least down to $10 \mathrm{~K}$ [18]. According to our results, the delta phases are thermodynamically more stable than this orthorhombic phase at $0 \mathrm{~K}$, and hence will become accessible at sufficiently low temperatures. However, the delta phases might be difficult to access since (a) the transition from orthorhombic symmetry to the delta phases is presumably a first-order transition (i.e., a discontinuous transition) based on connecting basins of symmetry [74,75] and (b) involves significant structural rearrangement of internal coordinates to form the relevant delta motifs with a presumably kinetic activation barrier that is unlikely to be overcome below $10 \mathrm{~K}$.

An issue that demands further investigation is whether the inclusion of zero-point energy (ZPE) and vibrational entropy influences the energetic ordering, i.e., if there is a strong entropic penalty of the delta phases with respect to the orthorhombic structure. However, addressing this issue is challenging since both harmonic and quasiharmonic phonon calculations lead to imaginary modes that arise from ill described Van der Waals interactions and volume effects [76]. It has been also established that the rotational modes of the organic $\mathrm{CH}_{3} \mathrm{NH}_{3}^{+}$cations are strongly anharmonic, and that there is a relatively large anharmonic coupling between the organic librational modes and the different optical modes of the octahedral framework [50]. Considering the large number of atoms in the double-delta $(\mathrm{B}, \mathrm{C})$ phases, advanced methods would be necessary to study the effects of anharmonicity in these novel phases. Indeed, vibrational entropy could in principle dominate the phase stability [77], but obtaining a conclusive picture to fully understand this issue in pervoskites is not trivial.

Further, the structures of the double-delta and delta phases are closely related to the $\mathrm{CsPbI}_{3}$, and the Yellow-FAPbI 
(formamidinium) phases, respectively [78-82]. Other closely related structures have been also derived from the scaffold mentioned before leading to stable hexagonal structure of MAPI [83] (both face and edge sharing).

Moreover, the effect of humidity on the degradation of perovskite (MAPI) solar cell performance also plays a significant role, and has been thoroughly studied in the past [84]. Thanks to those studies, it is well accepted that in the presence of water, $\mathrm{H}_{2} \mathrm{O}$ molecules deprotonates $\mathrm{CH}_{3} \mathrm{NH}_{3}^{+}$[85], thereby leading to product compounds with wider band gaps and substantially altered structural motifs, which are surprisingly similar to the double-delta phase (see Manser et al. Ref. [84]).

As discussed in detail in Sec. IIB, the enthalpy of the high temperature polymorphs (cubic and tetragonal) greatly increases with respect to the orthorhombic phase at high pressure (see Fig. 2). This finding is in agreement with the experimental evidence that suggests a transformation to a disordered (amorphous) phase $[20,86]$. The apparent reaction path suggests that, starting from the tetragonal or cubic phase, the pressure-induced amorphization occurs rapidly and is accelerated by entropic (temperature) effects. Szafrański-Katrusiak reported that the tetragonal phase $(I 4 / \mathrm{mcm})$ transforms via a first-order transition to a IV-phase with $\operatorname{Im} 3$ symmetry at $0.35 \mathrm{GPa}$, and above $2.5 \mathrm{GPa}$ either to a phase $\mathrm{V}(\mathrm{Im} 3)$ or to an amorphous phase [22]. Note that to correctly assign a crystal structure to the phases IV and V, a cell with 8 formula units (the same number of f.u. to describe the double-delta phase) is required. The same authors also list a range of possible sources of uncertainties that arise when performing single-crystal XRD measurements under pressure. In particular, the use of intense synchrotron beams for diffraction studies enhances potentially misleading effects: multiple scattering and higher-harmonics of $\lambda$ diffraction. Due to these technical difficulties, a plethora of different structures have been suggested that can be indexed to the same XRD spectrum, for instance Imm2, Pnma, Im3, Immm, or Fmmm have been assigned to the phases IV and V, with a degree of disorder of iodide ions and distorted structural parameters [24]. Other experiments even suggest metalization and stabilization of phases under a considerable amount of pressure [86].

Based on our careful analysis of the available literature work, none of the experimental high-pressure experiments used the low-temperature orthorhombic phase as the starting material. Instead, our simulated XRD patterns show that the tetragonal phases is present in the samples of Capitani [20], Jiang [23], and Jeffe [86] (See SI). Based on our calculations, we therefore suggest that either the delta or double delta phases could be synthesized by compressing precursor samples in the orthorhombic (Pnma) phase in low-temperature compression experiments. Since the orthorhombic phase is strongly destabilized upon compression, a transition towards the doubledelta or delta phases will be rapidly favored with increasing pressure. Such an approach based on cold-compression is often overlooked in materials synthesis, but has been shown in the past to be a promising method for stabilizing elemental phases in carbon and phosphorus [87-89].

Finally, we discuss the electronic properties of the MAPI phases. If they were synthesized in experiments, delta and double-delta both would exhibit very distinct electronic properties compared to any known phases. A corner-sharing octahe- dra (as in cubic, tetragonal and orthorhombic phases) promote a destabilization of the $s^{2}$ lone pairs on the $\mathrm{M}^{2+}$-sites, leading to very dispersive valence and conduction bands (high mobility of charge carriers). Additionally, they lead to band structures that exhibit direct band-gaps within 1.5-2.1 eV [82], a range that is suitable for many (opto-)electronic applications. Filip et al. [90] showed through an in silico study that the optical gap could be potentially tuned from the mid-infrared to the visible range via steric engineering. Even though this work is limited by the assumed Platonic model of $\mathrm{PbI}_{6}$, a rather restricted configurational landscape for perovskite materials, it was shown that the concept is applicable in a more general context [91] (delta and double delta models were not considered their works). For the (double) delta phases, the difference in the band gaps arises from the different connectivity or interoctahedral topology, namely edge- or face-sharing, where the lone pair is lowered in energy and leads to flatter electronic bands. Overall, accessing the new octahedral motifs observed in the (double) delta $\mathrm{PbI}_{6}$ would increase the design space to explore new organic and perovskite inorganic-frameworks. We suggest to further investigate synthetic routes based on compression and strain engineering to stabilize such structural features.

\section{CONCLUSION}

To summarize, we perform an in silico exploration of the structural landscape of hybrid organic-inorganic lead-iodide perovskites. We employ very large, realistic simulation cells with up to 96 atoms ( 8 formula units) for an extensive structural search, which allowed us to describe all the three known polymorphs on the same theoretical footing. From an exploration of more than 140,000 crystal structures, we identify novel low-energy phases with previously unobserved structural motifs for this system. The most promising candidates are the delta and double-delta phases, which are closely related to the reported yellow formamidinium non-perovskite phase and Cs lead iodides [79]. According to our DFT calculations at $0 \mathrm{~K}$, these delta-phases in MAPI are lower in energy than the experimentally observed orthorhombic phase. In contrast to any known phases of MAPI, the delta-phases exhibit edgeand surface sharing octahedral motifs, which in turn give rise to larger band gaps than perovskite phases. Different orientations of these octahedral moieties upon compression lead to changes in the band gaps. Hence, the electronic structure can be readily affected through phase selection and strain engineering, e.g., in thin-film synthesis.

\section{METHODS}

Unbiased configurational exploration of MAPI was performed with the minima hopping algorithm [33,34]. Energy and forces at the force field level were evaluated using the General Utility Lattice Program (GULP) molecular modeling package [36], employing force field parameters developed by Handley and Freeman [35]. Exploratory runs were conducted with simulation cells containing $12,24,48$, and 96 atoms at zero pressure.

Energies, atomic forces, and stresses at the density functional theory level were calculated with the VIENNA AB INITIO SIMULATION PACKAGE (VASP) [92]. Various approximations to the exchange-correlation functionals were tested, but the 
thermodynamic properties were computed using the strongly constrained and appropriately normed semilocal density functional meta-GGA (SCAN) [47]. A plane-wave basis set with a cutoff energy of $800 \mathrm{eV}$ was used within the projector augmented wave (PAW) formalism. The basis set was constructed with $\mathrm{Pb}$ d-shell (14), I (7), N (5), C (4), H (1) electrons as valence states in the PAW potentials. All calculations were spin unpolarized. The reciprocal space was sampled using $\Gamma$-centered $k$-grid meshes with spacings of $0.3 \AA^{-1}$. Geometry relaxations were performed with tight tolerance parameters, leading to atomic forces of less than $5 \mathrm{meV} / \AA$, and stresses below $0.1 \mathrm{eV} / \AA^{3}$.

\section{ACKNOWLEDGMENTS}

D.T. and S.G. thank C. Handley for his help with the MAPI forcefield. M.A. acknowledges support from the Novartis Universität Basel Excellence Scholarship for Life Sciences and the Swiss National Science Foundation (Project No. P300P2158407, No. P300P2-174475). Computational resources from the Swiss National Supercomputing Center (CSCS) in Lugano are gratefully acknowledged, J.A.F.-L. for project s752, D.T. and S.G. for project s707, M.A. for project s700, and U.R. and A.B. for projects 5789 and s672. This research was supported by the NCCR MARVEL, funded by the Swiss National Science Foundation.
[1] J. M. Buriak, P. V. Kamat, K. S. Schanze, A. P. Alivisatos, C. J. Murphy, G. C. Schatz, G. D. Scholes, P. J. Stang, and P. S. Weiss, Chem. Mater. 29, 8915 (2017).

[2] Y. Nishihata, J. Mizuki, T. Akao, H. Tanaka, M. Uenishi, M. Kimura, T. Okamoto, and N. Hamada, Nature 418, 164 (2002).

[3] F. Deschler, M. Price, S. Pathak, L. E. Klintberg, D.-D. Jarausch, R. Higler, S. Hüttner, T. Leijtens, S. D. Stranks, H. J. Snaith, M. Atatüre, R. T. Phillips, and R. H. Friend, J. Phys. Chem. Lett. 5, 1421 (2014).

[4] T. Okuda, K. Nakanishi, S. Miyasaka, and Y. Tokura, Phys. Rev. B 63, 113104 (2001).

[5] M. Liu, M. B. Johnston, and H. J. Snaith, Nature 501, 395 (2013).

[6] M. M. Lee, J. Teuscher, T. Miyasaka, T. N. Murakami, and H. J. Snaith, Science 338, 643 (2012).

[7] W. Eerenstein, N. D. Mathur, and J. F. Scott, Nature 442, 759 (2006).

[8] N. Hur, S. Park, P. A. Sharma, J. S. Ahn, S. Guha, and S.-W. Cheong, Nature 429, 392 (2004).

[9] A. P. Ramirez, J. Phys: Condens. Matter 9, 8171 (1997).

[10] M. A. Beno, L. Soderholm, D. W. Capone, II, D. G. Hinks, and J. D. Jorgensen, Appl. Phys. Lett. 51, 57 (1987).

[11] M. K. Wu, J. R. Ashburn, C. J. Torng, P. H. Hor, R. L. Meng, L. Gao, Z. J. Huang, Y. Q. Wang, and C. W. Chu, Phys. Rev. Lett. 58, 908 (1987).

[12] J. Haruyama, K. Sodeyama, L. Han, and Y. Tateyama, J. Phys. Chem. Lett. 5, 2903 (2014).

[13] Innovative Research and Products (iRAP) Inc. ET112: Piezoelectric Actuators and Motors-Types, Applications, new developments, Industry Structure and Global Markets (2010).

[14] R. H. Mitchell, Perovskites: Modern and Ancient (Almaz, Thunder Bay, Canada, 2002).

[15] A. Kojima, K. Teshima, Y. Shirai, and T. Miyasaka, J. Am. Chem. Soc. 131, 6050 (2009).

[16] F. Oba and Y. Kumagai, Appl. Phys. Express 11, 060101 (2018).

[17] H. N. Lee, H. M. Christen, M. F. Chisholm, C. M. Rouleau, and D. H. Lowndes, Nature 433, 395 (2005).

[18] P. Whitfield, N. Herron, W. Guise, K. Page, Y. Cheng, I. Milas, and M. Crawford, Sci. Rep. 6, 35685 (2016).

[19] A. Glazer, Acta Crystallogr., Sect. B: Struct. Crystallogr. Cryst. Chem. 28, 3384 (1972).

[20] F. Capitani, C. Marini, S. Caramazza, P. Postorino, G. Garbarino, M. Hanfland, A. Pisanu, P. Quadrelli, and L. Malavasi, J. Appl. Phys. 119, 185901 (2016).
[21] M. Szafranski and A. Katrusiak, J. Phys. Chem. Lett. 7, 3458 (2016).

[22] M. Szafrański and A. Katrusiak, J. Phys. Chem. Lett. 8, 2496 (2017).

[23] S. Jiang, Y. Fang, R. Li, H. Xiao, J. Crowley, C. Wang, T. J. White, W. A. Goddard, Z. Wang, T. Baikie, and J. Fang, Angew. Chem., Int. Ed. 55, 6540 (2016).

[24] A. Jaffe, Y. Lin, C. M. Beavers, J. Voss, W. L. Mao, and H. I. Karunadasa, ACS Cent. Sci. 2, 201 (2016).

[25] A. Jaffe, Y. Lin, and H. I. Karunadasa, ACS Energy Lett. 2, 1549 (2017).

[26] J. Even, L. Pedesseau, J.-M. Jancu, and C. Katan, J. Phys. Chem. Lett. 4, 2999 (2013).

[27] S. M. Korbel, S. Botti, and M. A. Marques, J. Mater. Chem. A 6, 6463 (2018).

[28] A. A. Emery, J.E. Saal, S. Kirklin, V. I. Hegde, and C. Wolverton, Chem. Mater. 28, 5621 (2016).

[29] J. Schmidt, J. Shi, P. Borlido, L. Chen, S. Botti, and M. A. Marques, Chemistry of Materials 29, 5090 (2017).

[30] R. Sarmiento-Perez, T. F. Cerqueira, S. Körbel, S. Botti, and M. A. Marques, Chemistry of Materials 27, 5957 (2015).

[31] T. D. Huan, V. N. Tuoc, and N. V. Minh, Phys. Rev. B 93, 094105 (2016).

[32] C. Kim, T. D. Huan, S. Krishnan, and R. Ramprasad, Sci. Data 4, 170057 (2017), data Descriptor.

[33] S. Goedecker, J. Chem. Phys. 120, 9911 (2004).

[34] M. Amsler and S. Goedecker, J. Chem. Phys. 133, 224104 (2010).

[35] C. M. Handley and C. L. Freeman, Phys. Chem. Chem. Phys. 19, 2313 (2017).

[36] J. D. Gale and A. L. Rohl, Mol. Simul. 29, 291 (2003).

[37] See Supplemental Material at http://link.aps.org/supplemental/ 10.1103/PhysRevMaterials.2.085201 for details on energy correlation between the force-field and DFT, structural fingerprint, electronic band gaps, simulated XRD pattern, and structural analysis of the Double-Delta phases.

[38] J. P. Perdew and Y. Wang, Phys. Rev. B 45, 13244 (1992).

[39] J. P. Perdew, K. Burke, and M. Ernzerhof, Phys. Rev. Lett. 77, 3865 (1996).

[40] J. P. Perdew, A. Ruzsinszky, G. I. Csonka, O. A. Vydrov, G. E. Scuseria, L. A. Constantin, X. Zhou, and K. Burke, Phys. Rev. Lett. 100, 136406 (2008).

[41] S. Grimme, J. Comput. Chem. 27, 1787 (2006). 
[42] S. Grimme, J. Antony, S. Ehrlich, and H. Krieg, J. Chem. Phys. 132, 154104 (2010).

[43] A. Tkatchenko and M. Scheffler, Phys. Rev. Lett. 102, 073005 (2009).

[44] A. Tkatchenko, R. A. DiStasio, Jr., R. Car, and M. Scheffler, Phys. Rev. Lett. 108, 236402 (2012).

[45] S. N. Steinmann and C. Corminboeuf, J. Chem. Phys. 134, 044117 (2011).

[46] C. Adamo and V. Barone, J. Chem. Phys. 110, 6158 (1999).

[47] J. Sun, R. C. Remsing, Y. Zhang, Z. Sun, A. Ruzsinszky, H. Peng, Z. Yang, A. Paul, U. Waghmare, X. Wu, M. L. Klein, and J. P. Perdew, Nat. Chem. 8, 831 (2016).

[48] J. Li and P. Rinke, Phys. Rev. B 94, 045201 (2016).

[49] Y. Wang, T. Gould, J. F. Dobson, H. Zhang, H. Yang, X. Yao, and H. Zhao, Phys. Chem. Chem. Phys. 16, 1424 (2014).

[50] M. A. Pérez-Osorio, A. Champagne, M. Zacharias, G.-M. Rignanese, and F. Giustino, J. Phys. Chem. C 121, 18459 (2017).

[51] J. Wiktor, U. Rothlisberger, and A. Pasquarello, J. Phys. Chem. Lett. 8, 5507 (2017).

[52] Y. Zhang, D. A. Kitchaev, J. Yang, T. Chen, S. T. Dacek, R. A. Sarmiento-Perez, M. A. Marques, H. Peng, G. Ceder, and J. P. Perdew, npj Comput. Mater. 4, 9 (2018).

[53] J. Lahnsteiner, G. Kresse, A. Kumar, D. D. Sarma, C. Franchini, and M. Bokdam, Phys. Rev. B 94, 214114 (2016).

[54] M. Bokdam, J. Lahnsteiner, B. Ramberger, T. Schäfer, and G. Kresse, Phys. Rev. Lett. 119, 145501 (2017).

[55] L. Zhu et al., J. Chem. Phys. 144, 034203 (2016).

[56] J. A. Flores-Livas, A. Sanna, and S. Goedecker, Novel Superconducting Materials 3, 6 (2017).

[57] W. Sun, S. T. Dacek, S. P. Ong, G. Hautier, A. Jain, W. D. Richards, A. C. Gamst, K. A. Persson, and G. Ceder, Sci. Adv. 2, e1600225 (2016).

[58] M. Amsler, V. I. Hegde, S. D. Jacobsen, and C. Wolverton, arXiv:1802.06900.

[59] H. Jagodzinski, Acta Crystallogr. 2, 201 (1949).

[60] R. Tilley, Perovskites: Structure-Property Relationships (Wiley, New York, 2016).

[61] See Supplemental Material at http://link.aps.org/supplemental/ 10.1103/PhysRevMaterials.2.085201 for more information.

[62] I. Swainson, M. Tucker, D. Wilson, B. Winkler, and V. Milman, Chem. Mat. 19, 2401 (2007).

[63] Y. Wang, X. Lü, W. Yang, T. Wen, L. Yang, X. Ren, L. Wang, Z. Lin, and Y. Zhao, J. Am. Chem. Soc. 137, 11144 (2015).

[64] P. Postorino and L. Malavasi, J. Phys. Chem. Lett. 8, 2613 (2017).

[65] P. Umari, E. Mosconi, and F. De Angelis, Sci. Rep. 4, 4467 (2014).

[66] J.-C. Blancon, H. Tsai, W. Nie, C. C. Stoumpos, L. Pedesseau, C. Katan, M. Kepenekian, C. M. M. Soe, K. Appavoo, M. Y. Sfeir, S. Tretiak, P. M. Ajayan, M. G. Kanatzidis, J. Even, J. J. Crochet, and A. D. Mohite, Science 355, 1288 (2017).

[67] M. I. Dar, G. Jacopin, S. Meloni, A. Mattoni, N. Arora, A. Boziki, S. M. Zakeeruddin, U. Rothlisberger, and M. Grätzel, Sci. Adv. 2, e1601156 (2016).

[68] G. E. Eperon, S. D. Stranks, C. Menelaou, M. B. Johnston, L. M. Herz, and H. J. Snaith, Energy \& Environmental Science 7, 982 (2014).

[69] T. Baikie, Y. Fang, J. M. Kadro, M. Schreyer, F. Wei, S. G. Mhaisalkar, M. Graetzel, and T. J. White, J. Mater. Chem. A 1, 5628 (2013).
[70] R. L. Milot, G. E. Eperon, H. J. Snaith, M. B. Johnston, and L. M. Herz, Adv. Funct. Mater. 25, 6218 (2015).

[71] C. Motta, F. El-Mellouhi, S. Kais, N. Tabet, F. Alharbi, and S. Sanvito, Nat. Commun. 6, 7026 (2015).

[72] A. M. A. Leguy, P. Azarhoosh, M. I. Alonso, M. Campoy-Quiles, O. J. Weber, J. Yao, D. Bryant, M. T. Weller, J. Nelson, A. Walsh, M. van Schilfgaarde, and P. R. F. Barnes, Nanoscale 8, 6317 (2016).

[73] H. Kim, J. Hunger, E. Cánovas, M. Karakus, Z. Mics, M. Grechko, D. Turchinovich, S. H. Parekh, and M. Bonn, Nat. Commun. 8, 687 (2017).

[74] J.-C. Toledano and P. Toledano, The Landau Theory of Phase Transitions: Application to Structural, Incommensurate, Magnetic and Liquid Crystal Systems (World Scientific, Singapore, 1987), Vol. 3.

[75] C. J. Howard and H. T. Stokes, Acta Crystallogr., Sect. B: Struct. Sci. 54, 782 (1998).

[76] F. Brivio, J. M. Frost, J. M. Skelton, A. J. Jackson, O. J. Weber, M. T. Weller, A. R. Goñi, A. M. A. Leguy, P. R. F. Barnes, and A. Walsh, Phys. Rev. B 92, 144308 (2015).

[77] G. Kieslich and A. L. Goodwin, Mater. Horiz. 4, 362 (2017).

[78] C. Yi, J. Luo, S. Meloni, A. Boziki, N. Ashari-Astani, C. Grätzel, S. M. Zakeeruddin, U. Röthlisberger, and M. Grätzel, Energy \& Environmental Science 9, 656 (2016).

[79] F. Ma, J. Li, W. Li, N. Lin, L. Wang, and J. Qiao, Chem. Sci. 8, 800 (2017).

[80] G. E. Eperon, G. M. Paterno, R. J. Sutton, A. Zampetti, A. A. Haghighirad, F. Cacialli, and H. J. Snaith, J. Mater. Chem. A 3, 19688 (2015).

[81] M. Lai, Q. Kong, C. G. Bischak, Y. Yu, L. Dou, S. W. Eaton, N. S. Ginsberg, and P. Yang, Nano Res. 10, 1107 (2017).

[82] C. C. Stoumpos, L. Mao, C. D. Malliakas, and M. G. Kanatzidis, Inorg. Chem. 56, 56 (2016)

[83] A. S. Thind, X. Huang, J. Sun, and R. Mishra, Chemistry of Materials 29, 6003 (2017).

[84] J. S. Manser, M. I. Saidaminov, J. A. Christians, O. M. Bakr, and P. V. Kamat, Acc. Chem. Res. 49, 330 (2016).

[85] J. M. Frost, K. T. Butler, F. Brivio, C. H. Hendon, M. Van Schilfgaarde, and A. Walsh, Nano Lett. 14, 2584 (2014).

[86] A. Jaffe, Y. Lin, W. L. Mao, and H. I. Karunadasa, J. Am. Chem. Soc. 139, 4330 (2017).

[87] W. L. Mao, H.-k. Mao, P. J. Eng, T. P. Trainor, M. Newville, C.-c. Kao, D. L. Heinz, J. Shu, Y. Meng, and R. J. Hemley, Science 302, 425 (2003).

[88] M. Amsler, J. A. Flores-Livas, L. Lehtovaara, F. Balima, S. A. Ghasemi, D. Machon, S. Pailhès, A. Willand, D. Caliste, S. Botti, A. San Miguel, S. Goedecker, and M. A. L. Marques, Phys. Rev. Lett. 108, 065501 (2012).

[89] J. A. Flores-Livas, A. Sanna, A. P. Drozdov, L. Boeri, G. Profeta, M. Eremets, and S. Goedecker, Phys. Rev. Mater. 1, 024802 (2017).

[90] M. R. Filip, G. E. Eperon, H. J. Snaith, and F. Giustino, Nat. Commun. 5, 5757 (2014).

[91] S. Meloni, G. Palermo, N. Ashari-Astani, M. Grätzel, and U. Rothlisberger, J. Mater. Chem. A 4, 15997 (2016).

[92] G. Kresse and J. Furthmüller, Comput. Mat. Sci. 6, 15 (1996). 